\title{
Isolation of Candida dubliniensis from denture wearers
}

\author{
Thaís Helena Gasparoto, ${ }^{1} \dagger$ Thiago José Dionísio, ${ }^{1} \dagger$ \\ Carine Ervolino de Oliveira, ${ }^{2}$ Vinicius Carvalho Porto, ${ }^{3}$ Valéria Gelani, ${ }^{1}$ \\ Carlos Ferreira Santos, ${ }^{1}$ Ana Paula Campanelli ${ }^{1}$ \\ and Vanessa Soares Lara ${ }^{2}$ \\ ${ }^{1}$ Department of Biological Sciences, Bauru School of Dentistry, University of São Paulo, Bauru, SP, \\ Brazil \\ ${ }^{2}$ Department of Stomatology (Pathology), Bauru School of Dentistry, University of São Paulo, Bauru, \\ $\mathrm{SP}$, Brazil \\ ${ }^{3}$ Department of Prosthodontics, Bauru School of Dentistry, University of São Paulo, Bauru, SP, \\ Brazil
}

Correspondence

Vanessa Soares Lara

vanessa@fob.usp.br

Received 30 November 2008

Accepted 4 March 2009
Candida albicans is considered the most important Candida species able to cause oral infections in denture wearers. In recent years, Candida dubliniensis has emerged as a pathogenic yeast in humans. The close phenotypic similarities of C. albicans and C. dubliniensis have led to the misidentification of these species. In this work, our aim was to verify through PCR the presence of C. dubliniensis in palate and maxillary denture samples from 112 denture wearers presenting with or without denture-related stomatitis (DRS). C. dubliniensis was isolated at low rates from both palate $(5.3 \%$ and $10.7 \%)$ and maxillary denture (5.3\% and $8.9 \%)$ samples from wearers regardless of the presence of the disease. However, when C. dubliniensis was detected in individuals with DRS, it was always associated with C. albicans. In addition, our results showed that $C$. albicans was the most commonly identified candidal species in maxillary denture and hard palate samples from DRS patients (78.5\% and $89.2 \%$, respectively) as well as from controls (31.2\% and $28.5 \%$, respectively). In conclusion, C. dubliniensis was detected in the oral environment of denture wearers. The association of C. dubliniensis with C. albicans occurred in approximately $10 \%$ of the DRS cases.

\section{INTRODUCTION}

Denture-related stomatitis (DRS) is a local recurring disease (Budtz-Jorgensen et al., 1975; Dar-Odeh \& Shehabi, 2003) that attacks the mucosal area subjacent to dental prostheses, beneath the fitting surface of the denture, especially maxillary dentures. Many studies have discussed the involvement of Candida albicans in the establishment and persistence of such disease since the acrylic denture fitting surfaces seem to facilitate the adherence of this micro-organism (Webb et al., 1998; Makihira et al., 2002; Ramage et al., 2004; Moura et al., 2006). Non-albicans Candida species can also be obtained from DRS lesions (Figueiral et al., 2007).

Recently, a newly identified Candida species has emerged, Candida dubliniensis, which exhibits numerous characteristics typical of $C$. albicans species, including the formation

†These authors contributed equally to this paper.

Abbreviation: DRS, denture-related stomatitis. of green colonies on CHROMagar medium. Both microorganisms share several phenotypic traits such as the ability to form true germ tubes, adhere to epithelial surfaces, secrete a range of aspartic proteinases and form chlamydospores (Martinez et al., 2002; Sullivan \& Coleman, 1998; Sahand et al., 2005; Gilfillan et al., 1998). C. dubliniensis was first obtained from oropharyngeal lesions of human immunodeficiency virus (HIV)-positive patients (Sullivan \& Coleman, 1998). Diabetic HIV-negative individuals also exhibited a high prevalence of C. dubliniensis in oral carriage and disease states (Willis et al., 2000). Recent work has documented the presence of $C$. dubliniensis in just one case of DRS lesion (Mosca et al., 2005). The association between C. dubliniensis and DRS suggests that this microorganism may play important roles in the establishment and persistence of DRS.

Therefore, in this work, we examined the presence of $C$. dubliniensis in samples from palate and maxillary dentures from 112 denture wearers presenting with or without DRS. In order to address this, CHROMagar culture and PCR 
were utilized. Our data demonstrated that Candida species were more frequently isolated from the palate as well as the internal surface of maxillary dentures from individuals with DRS than from control denture wearer individuals. PCR analysis indicated that C. dubliniensis was recovered from the palate and/or maxillary denture from both groups studied $(5.3-10.7 \%)$ and confirmed that C. albicans was the species most associated with the disease $(89.2 \%$ on palate and $78.5 \%$ on maxillary denture).

\section{METHODS}

Subject population. One hundred and twelve denture wearers were selected from the population referred to the Clinics of Prosthodontics at the Bauru School of Dentistry, University of São Paulo (Bauru, SP, Brazil). A complete clinical examination was performed, including medical and dental histories and an intra-oral examination. Fifty-six patients were clinically diagnosed as having DRS and 56 were designated healthy controls. The age of the participants ranged from 44 to 94 years. The protocol for all the procedures was approved by the Institutional Ethics Committee. All the subjects signed an informed consent.

Inclusion criteria were as follows: participants had to be complete acrylic (heat-cured resin) denture wearers, presenting with and without DRS, and who reported wearing their complete maxillary dentures for at least $12 \mathrm{~h}$ a day over the previous 2 years. The exclusion criteria were: smoking, alcoholism, types 1 and 2 diabetes, any diseases or medicines generating immunosuppression, autoimmune diseases and drug use (including antifungal medication).

Identification of DRS and Candida species. DRS associated with the palate was diagnosed as pinpoint hyperaemia, diffuse hyperaemia or granular, as reported by Newton (1962). The microbiological diagnosis was carried out by collecting material from the hard palate in a denture stomatitis-associated erythematous area, from underneath the denture and from the surface of dentures in contact with the mucosa with a sterile swab. The samples from the control group were obtained from the denture fitting surface and hard palate covered by the maxillary denture. All samples were grown on Sabouraud dextrose broth (Difco, Becton Dickinson) with $1 \%$ chloramphenicol (Sigma-Aldrich) at room temperature. Such a step was completed in order to avoid false-negativity for the presence of Candida and therefore c.f.u. were not counted. After 1 week, broth along with samples was inoculated onto CHROMagar Candida plates (Becton Dickinson) to identify Candida species. CHROMagar Candida allowed identification of C. albicans, Candida tropicalis and Candida krusei through the growth of yeast colonies showing different colours and morphologies (Pfaller et al., 1996; Willinger et al., 2001). Samples grown on CHROMagar Candida that displayed a dark- or light-green colour were selected.

DNA extraction. The modified technique of Romeo et al. (2006) to differentiate C. albicans and C. dubliniensis was used. After the yeast samples were grown on CHROMagar plates, green colonies were obtained and maintained on Sabouraud dextrose agar at room temperature until DNA purification. Yeasts were washed three times at $10000 \mathrm{~g}$ for $5 \mathrm{~min}$ at $4{ }^{\circ} \mathrm{C}$. The pellet was suspended in $0.1 \mathrm{ml}$ DNase/Rnase-free water $+0.1 \mathrm{ml}$ InstaGene (InstaGene Matrix; BioRad Laboratories) and incubated for $30 \mathrm{~min}$ at $56{ }^{\circ} \mathrm{C}$. The samples were then boiled for $10 \mathrm{~min}$ and centrifuged at $10000 \mathrm{~g}$ for $5 \mathrm{~min}$ at $4{ }^{\circ} \mathrm{C}$ (Sakai et al., 2007). Supernatants were obtained for the analysis of C. albicans or C. dubliniensis DNA.
PCR amplification of C. albicans and C. dubliniensis. Samples were amplified under standardized conditions using a species-specific primer set for the hyphal wall protein 1 gene (CRR forward primer, 5'-GTTTTTGCAACTTCTCTTTGTTTA-3'; and CRR reverse primer, $5^{\prime}$-ACAGTTGTATCATGTTCAGT- $3^{\prime}$ ). The PCR mixture (total volume $25 \mu \mathrm{l}$ ) contained $3 \mu \mathrm{l}$ genomic DNA template, $0.2 \mathrm{mM}$ each deoxynucleoside triphosphate (IDT primer), $0.4 \mu \mathrm{M}$ each primer, $2.5 \mathrm{U}$ GoTaq polymerase (Promega) and $1.5 \mathrm{mM} \mathrm{MgCl}_{2}$. Amplification was performed after denaturation at $95{ }^{\circ} \mathrm{C}$ for $5 \mathrm{~min}$ followed by 34 cycles of denaturation at $94{ }^{\circ} \mathrm{C}$ for $45 \mathrm{~s}$, primer annealing at $50{ }^{\circ} \mathrm{C}$ for $60 \mathrm{~s}$, and an extension at $72{ }^{\circ} \mathrm{C}$ for $90 \mathrm{~s}$, followed by a final extension at $72{ }^{\circ} \mathrm{C}$ for $10 \mathrm{~min}$ in a thermocycler (Progene; Techne).

PCR products were separated on a $1.5 \%(\mathrm{w} / \mathrm{v})$ agarose gel, stained with ethidium bromide $\left(0.5 \mu \mathrm{g} \mathrm{ml}^{-1}\right)$, and compared with the DNA size marker (100 Base-Pair Ladder; Amersham Biosciences) using a transilluminator (Sigma T2202). Two different amplicons were expected: $1180 \mathrm{bp}$ for C. albicans and $930 \mathrm{bp}$ for C. dubliniensis.

Statistical analysis. Data were analysed with GraphPad Prism version 5.00 for Windows (www.graphpad.com), using the chi-square test (with Yates' correction) and Fisher's exact test, to compare the proportion of each variable in the groups with or without DRS and where yeasts were identified or not. Values of $P<0.05$ were considered statistically significant.

\section{RESULTS AND DISCUSSION}

DRS is the major oral infection in elderly denture wearers, and C. albicans is the micro-organism mostly involved in this disease (Budtz-Jorgensen et al., 1975; Dar-Odeh \& Shehabi, 2003; Webb et al., 1998; Figueiral et al., 2007). Clinical evaluation showed that type I DRS was detected most often in our patients, followed by type II and type III $(n=36,17$ and 3, respectively; Table 1). Our data are in accordance with those of Figueiral et al. (2007). Regardless of the DRS type, Candida species were present in almost all of the cases (Table 1). Also, a higher occurrence of Candida species was found in the palate and maxillary denture samples from DRS patients than in samples from the control individuals (Table 1).

We further investigated which Candida species were present in individuals presenting with and without DRS through CHROMagar Candida. Our results showed that $C$. krusei and C. tropicalis were cultivated from patients with and without DRS. However, an association of different Candida species (C. krusei, C. tropicalis and C. albicans) on the hard palate mucosa and internal surface maxillary denture was verified only in DRS patients (data not shown). The importance of interactions between Candida species in biofilm formation has been extensively studied (Thein et al., 2007a, b). However, further studies are necessary to understand the role of non-albicans Candida species in DRS as well as in the absence of the disease.

The colonies that were identified as albicans (green colonies) by CHROMagar Candida were submitted to molecular analysis by PCR in order to investigate the presence of $C$. dubliniensis in such samples. Our data revealed that C. dubliniensis was obtained in $10.7 \%(n=6)$ 
Table 1. Demographic, microbiological and clinical data obtained from subjects with and without DRS

\begin{tabular}{|llcl|}
\hline Variable & DRS patients $(\boldsymbol{n}=\mathbf{5 6})$ & Healthy controls $(\boldsymbol{n}=\mathbf{5 6})$ & $\boldsymbol{P}$-value \\
\hline Age (mean \pm standard deviation) & $69.4 \pm 9.2$ & $68.2 \pm 5.5$ & 0.9 \\
Female/male ratio & $39 / 17$ & $34 / 22$ & 0.99 \\
Candida species on maxillary denture $(n)$ & $98.2 \%(55)$ & $44.7 \%(25)$ & $0.01^{\star}$ \\
Candida species on palate $(n)$ & $98.2 \%(55)$ & $42.9 \%(24)$ & $0.01^{\star}$ \\
Type of lesion $(n)$ & $64.2 \%$, type I (36) & Not present & \\
& $30.3 \%$, type II $(17)$ & & \\
& $5.3 \%$, type III (3) & & \\
\hline
\end{tabular}

*Statistically significant difference comparing Candida species on maxillary denture or Candida species on palate in both groups.

of palate and $8.9 \%(n=5)$ of denture samples from DRS patients, and in $5.3 \%(n=3)$ of both palate and maxillary denture samples from controls. Although a slight difference was verified for $C$. dubliniensis detection between DRS patients and controls, it was not statistically significant $(P=0.09)$. However, there was a significantly higher percentage of C. albicans in palate $(89.2 \%)$ and maxillary denture $(78.5 \%)$ samples from patients with DRS in comparison with controls $(28.5 \%$ and $31.2 \%$, respectively; $P<0.05)$. In fact, DRS has been suggested to occur due to the outgrowth of $C$. albicans commensal strains (Mathaba et al., 1995). Interestingly, all maxillary denture and palate samples from DRS patients that contained C. dubliniensis also contained C. albicans.

Another study identified $C$. dubliniensis in palate mucosa and denture samples from a patient with DRS; however, this micro-organism was not found in individuals without DRS (Marcos-Arias et al., 2009). Together, our results show the presence of $C$. dubliniensis in complete maxillary denture wearers regardless of the presence of DRS. Therefore, our data could suggest that $C$. dubliniensis does not have an essential role in the pathogenesis of DRS.

The presence of $C$. dubliniensis has been more frequently detected in individuals with systemic metabolic alterations, i.e. symptomatic HIV-positive and diabetic patients (Sullivan et al., 1995; Sullivan \& Coleman, 1997, 1998; Kirkpatrick et al., 1998; Martinez et al., 2002). However, C. dubliniensis has been shown to present physiological and phenotypic traits similar to those of $C$. albicans, and this constitutes an important virulence factor for its pathogenicity (Moran et al., 1997; Gilfillan et al., 1998; Ramage et al., 2001; Shin et al., 2002). In addition, the treatment of patients with some antifungal drugs has generated the replacement of C. albicans by C. dubliniensis (Martinez et al., 2002). Our control and DRS patients had not been given any antifungal therapy and perhaps this might explain why they maintained concomitantly C. dubliniensis and $C$. albicans colonizing their palate and maxillary denture.

Future studies will be necessary to understand the influence of C. dubliniensis in the oral environment of the systemically normal population, including denture wearers.

\section{ACKNOWLEDGEMENTS}

This work was supported by FAPESP (05/60668-9 and 06/59612-1).

\section{REFERENCES}

Budtz-Jorgensen, E., Stenderup, A. \& Grabowski, M. (1975). An epidemiologic study of yeasts in elderly denture wearers. Community Dent Oral Epidemiol 3, 115-119.

Dar-Odeh, N. S. \& Shehabi, A. A. (2003). Oral candidosis in patients with removable dentures. Mycoses 46, 187-191.

Figueiral, M. H., Azul, A., Pinto, E., Fonseca, P. A., Branco, F. M. \& Scully, C. (2007). Denture-related stomatitis: identification of aetiological and predisposing factors - a large cohort. J Oral Rehabil 34, 448-455.

Gilfillan, G. D., Sullivan, D. J., Haynes, K., Parkinson, T., Coleman, D. C. \& Gow, N. A. R. (1998). Candida dubliniensis: phylogeny and putative virulence factors. Microbiology 144, 829-838.

Kirkpatrick, W. R., Revankar, S. G., McAtee, R. K., Lopez-Ribot, J. L., Fothergill, A. W., McCarthy, D. I., Sanche, S. E., Cantu, R. A., Rinaldi, M. G. \& Patterson, T. F. (1998). Detection of Candida dubliniensis in oropharyngeal samples from human immunodeficiency virusinfected patients in North America by primary CHROMagar Candida screening and susceptibility testing of isolates. J Clin Microbiol 36, 3007-3012.

Makihira, S., Nikawa, H., Nishimura, M., Egusa, H., Sadamori, S., Rahayu, R. P., Nishimura, H. \& Hamada, T. (2002). Impact of components of denture acrylic resin on gingival cell growth and sensitivity to Candida albicans adhesion. Mycoses 45, 300-305.

Marcos-Arias, C., Vicente, J. L., Sahand, I. H., Eguia, A., De-Juan, A., Madariaga, L., Aguirre, J. M., Eraso, E. \& Quindós, G. (2009). Isolation of Candida dubliniensis in denture stomatitis. Arch Oral Biol 54, 127-131.

Martinez, M., López-Ribot, J. L., Kirkpatrick, W. R., Coco, B. J., Bachmann, S. P. \& Patterson, T. F. (2002). Replacement of Candida albicans with $C$. dubliniensis in human immunodeficiency virusinfected patients with oropharyngeal candidiasis treated with fluconazole. J Clin Microbiol 40, 3135-3139.

Mathaba, L. T., Davies, G. \& Warmington, J. R. (1995). The genotypic relationship of Candida albicans strains isolated from the oral cavity of patients with denture stomatitis. J Med Microbiol 42, 372379.

Moran, G. P., Sullivan, D. J., Henman, M. C., Mccreary, C. E., Harrington, B. J., Shanley, D. B. \& Coleman, D. C. (1997). Antifungal drug susceptibilities of oral Candida dubliniensis isolates from human immunodeficiency virus (HIV)-infected and non-HIV-infected sub- 
jects and generation of stable fluconazole-resistant derivatives in vitro. Antimicrob Agents Chemother 41, 617-623.

Mosca, C. O., Moragues, M. D., Brena, S., Rosa, A. C. \& Pontón, J. (2005). Aislamiento de Candida dubliniensis en un adolescente con estomatitis protésica. Med Oral Patol Oral Cir Bucal 10, 25-31.

Moura, J. S., da Silva, W. J., Pereira, T., Del Bel Cury, A. A. \& Garcia, R. C. R. (2006). Influence of acrylic resin polymerization methods and saliva on the adherence of four Candida species. J Prosthet Dent 96, 205-211.

Newton, A. V. (1962). Denture sore mouth as possible etiology. $\mathrm{Br}$ Dent J 112, 357-360.

Pfaller, M. A., Houston, A. \& Coffmann, S. (1996). Application of CHROMagar Candida for rapid screening of clinical specimens for Candida albicans, Candida tropicalis, Candida krusei, and Candida (Torulopsis) glabrata. J Clin Microbiol 34, 58-61.

Ramage, G., Vande Walle, K., Wickes, B. L. \& López-Ribot, J. L. (2001). Biofilm formation by Candida dubliniensis. J Clin Microbiol 39, 3234-3240.

Ramage, G., Tomsett, K., Wickes, B. L., López-Ribot, J. L. \& Redding, S. W. (2004). Denture stomatitis: a role for Candida biofilms. Oral Surg Oral Med Oral Pathol Oral Radiol Endod 98, 53-59.

Romeo, O., Racco, C. \& Criseo, G. (2006). Amplification of the hyphal wall protein 1 gene to distinguish Candida albicans from Candida dubliniensis. J Clin Microbiol 44, 2590-2592.

Sahand, I. H., Moragues, M. D., Eraso, E., Villar-Vidal, M., Quindós, G. \& Pontón, J. (2005). Supplementation of CHROMagar Candida medium with Pales medium for rapid identification of Candida dubliniensis. J Clin Microbiol 43, 5768-5770.

Sakai, V. T., Campos, M. R., Machado, M. A. A. M., Lauris, J. R. P., Greene, A. S. \& Santos, C. F. (2007). Prevalence of four putative periodontopathic bacteria in saliva of a group of Brazilian children with mixed dentition: 1year longitudinal study. Int J Paediatr Dent 17, 192-199.
Shin, J. H., Kee, S. J., Shin, M. G., Kim, S. H., Shin, D. H., Lee, S. K., Suh, S. P. \& Ryang, D. W. (2002). Biofilm production by isolates of Candida species recovered from nonneutropenic patients: comparison of bloodstream isolates with isolates from other sources. J Clin Microbiol 40, 1244-1248.

Sullivan, D. \& Coleman, D. (1997). Candida dubliniensis: an emerging opportunistic pathogen. Curr Top Med Mycol 8, 15-25.

Sullivan, D. \& Coleman, D. (1998). Candida dubliniensis: characteristics and identification. J Clin Microbiol 36, 329-334.

Sullivan, D. J., Westerneng, T. J., Haynes, K. A., Bennett, D. E. \& Coleman, D. C. (1995). Candida dubliniensis sp. nov.: phenotypic and molecular characterization of a novel species associated with oral candidosis in HIV-infected individuals. Microbiology 141, 1507-1521.

Thein, Z. M., Samaranayake, Y. H. \& Samaranayake, L. P. (2007a). In vitro biofilm formation of Candida albicans and non-albicans Candida species under dynamic and anaerobic conditions. Arch Oral Biol 52, 761-767.

Thein, Z. M., Samaranayake, Y. H. \& Samaranayake, L. P. (2007b). Characteristics of dual species Candida biofilms on denture acrylic surfaces. Arch Oral Biol 52, 1200-1208.

Webb, B. C., Thomas, C. J., Willcox, M. D. P., Harty, D. W. S. \& Knox, K. W. (1998). Candida-associated denture stomatitis: aetiology and management: a review. II. Oral diseases caused by Candida species. Aust Dent J 43, 160-166.

Willinger, B., Hillowoth, C., Selitsch, B. \& Manafi, M. (2001). Performance of Candida ID, a new chromogenic medium for presumptive identification of Candida species in comparison to CHROMagar Candida. J Clin Microbiol 39, 3793-3795.

Willis, A. M., Coulter, W. A., Sullivan, D. J., Coleman, D. C., Hayes, J. R., Bell, P. M. \& Lamey, P. J. (2000). Isolation of C. dubliniensis from insulin-using diabetes mellitus patients. J Oral Pathol Med 29, $86-90$. 